\title{
Feline arterial thromboembolism: prognostic factors and treatment
}

\author{
Feliene arteriële trombo-embolie: prognostische factoren en behandeling
}

${ }^{1}$ L. Locquet, ${ }^{1}$ D. Paepe, ${ }^{1}$ S. Daminet, ${ }^{1}$ P. Smets

${ }^{1}$ Small Animal Department, Faculty of Veterinary Medicine, Ghent University, Salisburylaan 133, 9820 Merelbeke, Belgium

Laurent.Locquet@ugent.be

\begin{abstract}
$\Lambda_{\text {bstract }}$
Feline arterial thromboembolism (ATE) is a complete or partial obstruction of a peripheral artery caused by a thrombus that was formed at a distant site. The most common underlying cause in cats is cardiomyopathy. Given the clinical presentation, often without preceding signs, an ATE event is considered one of the most distressing emergency conditions in feline practice. Often, these cats are euthanized at the time of diagnosis. However, recent scientific research has shown that a subpopulation of these patients may have long-term survival. In case of adequate treatment and follow-up, some cats survive over a year with a satisfying quality of life. Key points of ATE are the identification of specific prognostic factors in the individual patient in order to guide owner communication, the decision to treat or not to treat, individually adjusted patient management and regular monitoring, which are discussed in this article.
\end{abstract}

\section{SAMENVATTING}

Feliene arterïele trombo-embolie (ATE) wordt veroorzaakt door een volledige of gedeeltelijke obstructie van een perifere arterie ten gevolge van trombus die gevormd werd op een andere plaats; dit vaak ten gevolge van cardiomyopathie. Gezien de klinische presentatie, waaraan vaak geen symptomen vooraf gaan, wordt ATE beschouwd als een van de meest verontrustende, spoedeisende situaties in de praktijk. Een aanzienlijk deel van deze patiënten wordt geëuthanaseerd op het moment van diagnose. Recent wetenschappelijk onderzoek heeft echter aangetoond dat sommige van deze patiënten een goede langetermijnoverleving kunnen hebben. Deze katten kunnen soms tot langer dan één jaar overleven met een goede levenskwaliteit, mits een correcte behandeling en nauwkeurige opvolging. Essentieel bij deze aandoening is het herkennen van specifieke prognostische factoren om de communicatie met de eigenaar te sturen en zo in samenspraak de beslissing te nemen het dier te behandelen of te euthanaseren. Verder zijn een individueel aangepaste therapie en gerichte follow-up van de patiënt belangrijk.

\section{INTRODUCTION}

In feline practice, the occurrence of arterial thromboembolism (ATE) is one of the most distressing emergency situations for the cat, the client and the veterinarian. Currently, in first-line practice, these patients are commonly euthanized at the moment of diagnosis (Borgeat et al., 2014). Affected patients are usually presented with severe pain and associated vocalization, often combined with paralysis of the pelvic limbs and dyspnea or tachypnea. The most common form of ATE, the so-called "saddle thrombus", is caused by occlusion at the level of the distal aortic trifurcation. Diagnosis is primarily based on physical examination, with the typical clinical signs of pain, pulselessness, paralysis/paresis, pallor and poikilothermy, also referred to as the 5 P's. As an underlying cardiomyopathy is the most prevalent etiological cause, it is vital to recognize the presence of possible concomitant congestive heart failure (CHF), as this will influence both further medical management and prognosis. On auscultation, the presence of inspiratory crackles, a gallop rhythm or a murmur is highly suggestive of the presence of concomitant CHF. In general, the first 
treatment step for these painful and often tachypneic/ dyspneic cats consists of providing adequate pain relief medication and a stress-free environment with oxygen supplementation. Further investigations may be undertaken once the cat's pain, hypercoagulable state and possible CHF have been addressed via analgetic, antithrombotic and diuretic treatment. Although many of these cats are euthanized at the time of diagnosis, a correct identification of specific prognostic factors obtained from results of physical examination and further testing, enables the veterinarian to give the owner the best possible advice regarding further treatment, management and prognosis. The owner should be informed thoroughly about in hospital treatment and daily home medical care, the risk of ATE recurrence, presence of underlying cardiomyopathy, the need for monitoring, and associated costs. In this review, the pathophysiology of ATE is briefly explained to better understand clinical signs and diagnosis, with focus on prognostic factors aiding in the decision to treat, and different treatment options are discussed.

\section{PATHOPHYSIOLOGY}

\section{Physiological thrombogenesis}

In a healthy individual, thrombus formation is coordinated via interacting signalling pathways between platelets, coagulation factors and the endothelium, resulting in hemostasis without the risk of inappropriate thrombosis. Under physiological conditions, an intact endothelium normally produces antithrombotic factors, which help to maintain thromboresistance, whilst a damaged endothelial surface will trigger the coagulation cascade (Kapur et al., 2007). Furthermore, platelets will be activated via alternative pathways as well. The combination of these divergent pathways lead to a growing platelet plug with numerous platelets bridged together by fibrinogen. Initially, the thrombus is platelet-rich but quickly becomes fibrin-rich as the clot grows and matures (Helenski and Ross, 1985; Furie and Furie, 2008; Goggs and Poole, 2012).

\section{Arterial thromboembolism}

The thrombus becomes lamellated as it ages and superficial portions can break off, or more rarely, it dislodges in entirety, forming the emboli that travel to distant sites where their size eventually exceeds vessel diameter, resulting in infarction of an arterial bed (Collet, 1930). The thrombus causes a (partial) block of the obstructed artery and induces vasoconstriction of the collaterals, reducing perfusion and oxygenation of the tissue distal to the obstructed vessel. In cats with cardiomyopathy, the left auricular appendage is usually the site of thrombus formation. Certain arteries, e.g. the distal aorta, are more prone to obstruction, due to their anatomical conformation (Figure 1).

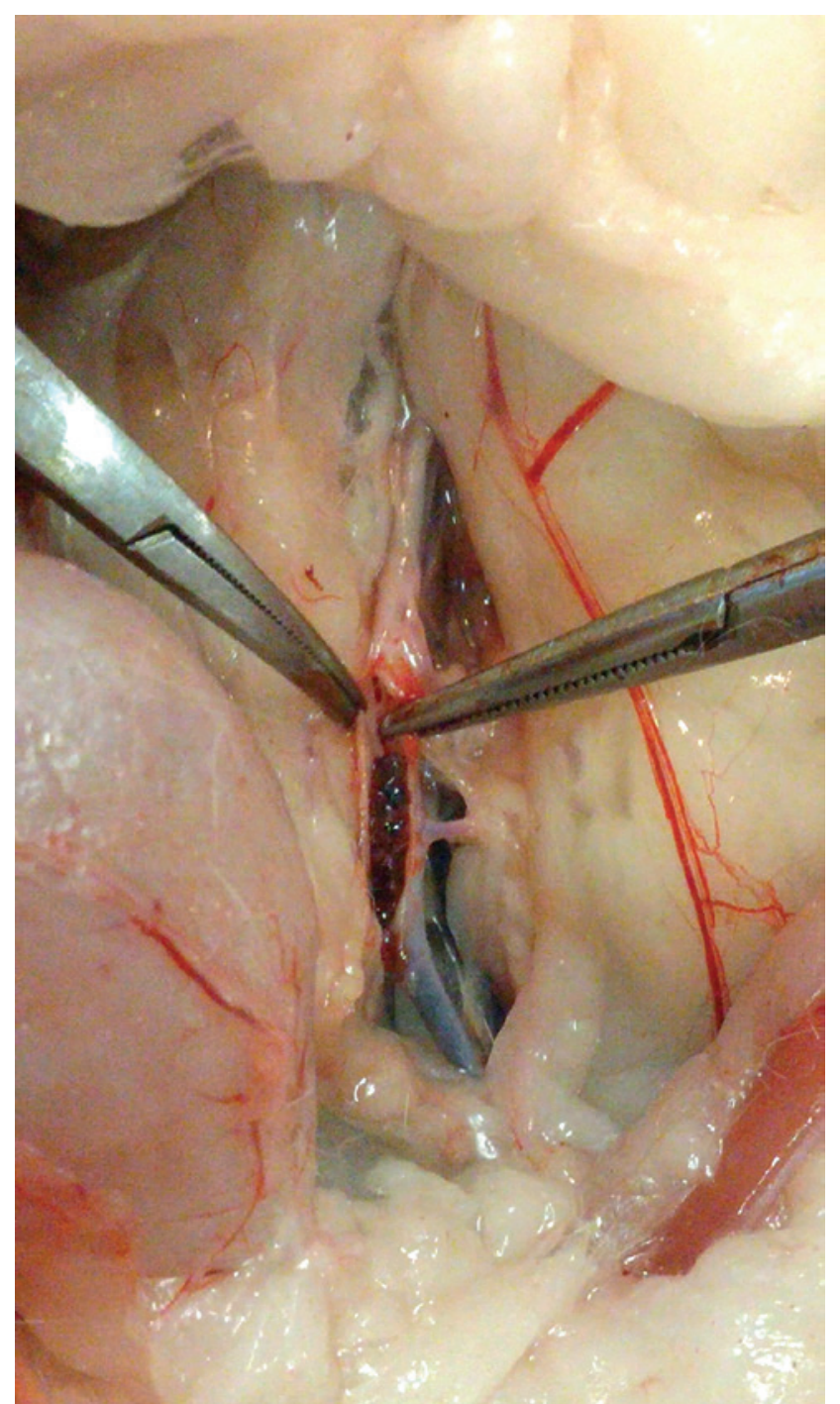

Figure 1. A thrombus obstructing the distal aorta at the level of the bifurcation (Photograph courtesy of $P$. Smets).

The most common underlying cause of thrombogenesis leading to an ATE event, is cardiac disease, and in the feline population, this is most commonly (in approximately 69\% of the cases) cardiomyopathy (Smith et al., 2004). Feline cardiomyopathy predisposes to formation of intracavitary thrombi due to blood stasis, endothelial injury and a hypercoagulable state, fulfilling all components of the Virchow triad (Welles et al., 1994; Schober and Maerz, 2006; Stokol et al., 2008).

\section{Blood stasis}

Blood stasis is primarily seen in the left atrium, when it is dilated secondary to the underlying cardiac disease. Low blood flow velocities have been linked to spontaneous echo contrast or 'smoke' in the left atrium and left auricular appendage, which can easily be detected using echocardiography (Figure 2). 


\section{Endothelial function}

Secondary to left atrium enlargement, increased intra-atrial pressure and stretch have been associated with endothelial injury, exposing subendothelial collagen or causing endothelial dysfunction, which facilitates platelet adhesion with subsequent activation and aggregation (Laste and Harpster, 1995; Smith et al., 2003; Furie and Furie, 2008).

\section{Platelet function}

The presence of a hypercoagulable state in cats is controversial, but the clinical occurrence of thrombosis has been associated with platelet hypersensitivity, increased procoagulantia and decreased antithrombin levels (Jandrey et al., 2008; Brazzell and Borjesson, 2007). However, neither left atrial size nor CHF were associated with hypercoagulability in a study by Stokol et al. (2008). This suggests that a subset of cats with cardiomyopathy may be hypercoagulable and develop ATE irrespective of atrial enlargement (Welles et al., 1994; Stokol et al., 2008).

Having discussed Virchow's triad and its different components in association with the pathogenesis of arterial thromboembolism, it can be concluded that an ATE event is a multifactorial process, with the most important factor being blood flow stasis associated with a dilated left atrium; other contributing factors, such as endothelial damage or hypercoagulability, may be involved to a lesser extent (Fuentes, 2012).

\section{PREVALENCE AND SIGNALMENT}

In several studies, divergent prevalence numbers have been shown, ranging from $0.6 \%$ in referral populations to $0.3 \%$ in general practice (Smith et al., 2003; Borgeat et al., 2014). In different studies, an ATE prevalence has been reported from 12 up to $21 \%$ in cats that have been diagnosed with HCM (Rush et al., 2000). Tomcats are overrepresented. This might reflect the male predisposition to cardiomyopathy, which is hence likely to be gender biased. There is no real breed predisposition, purebred cats are affected as well as domestic short- and longhair cats (Baty et al., 2001; Ferasin et al., 2003). Age at presentation with ATE ranges from one to twenty years, but the median age is eight to nine years (Laste and Harpster, 1995).

\section{HISTORY AND CLINICAL SIGNS}

Affected patients are usually presented with severe pain and associated vocalization, often combined with paralysis of the pelvic limbs and dyspnea or tachypnea. Because the trifurcation of the distal aorta is the most prevalent site of obstruction, an ATE event often leads to paresis or paralysis of the pelvic limbs due to ischemic neuromyopathy, although one leg might be more affected than the other. Infarction of one tho-

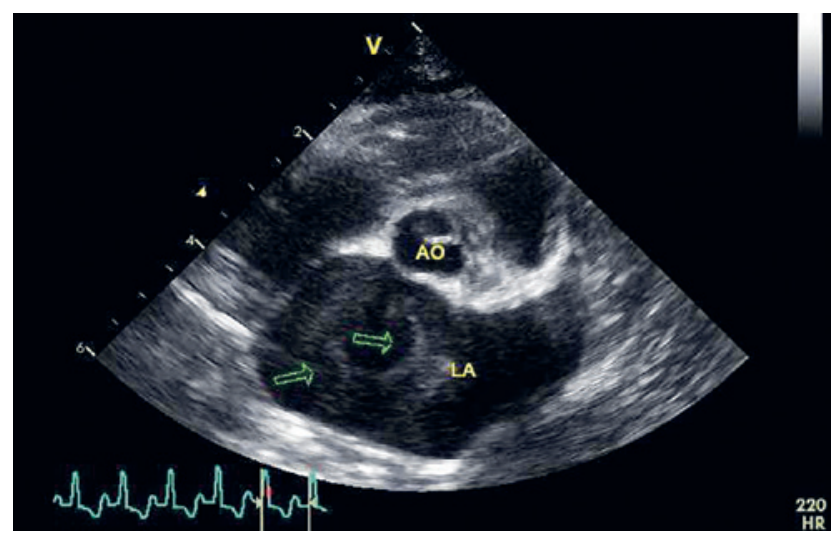

Figure 2. Spontaneous echocardiographic contrast, also known as 'smoke', can be distinguished within the enlarged left atrium (arrows) (LA: left atrium; Ao: aorta) (Photograph courtesy of P. Smets).

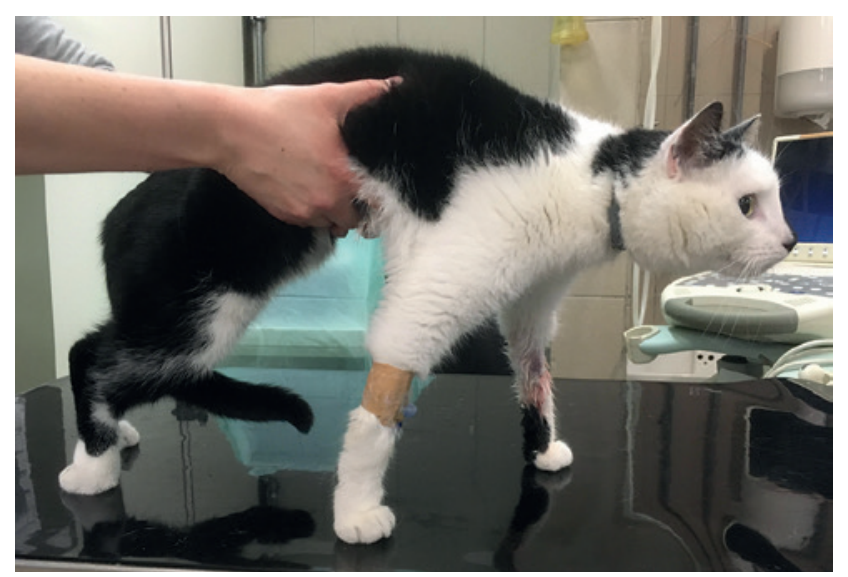

Figure 3. Abnormal stance (knuckling of the metatarsus) with paralysis of the right hind limb due to ATE (Photograph courtesy of P. Smets).

racic limb is the second most common clinical presentation of ATE in cats, with reported frequencies of 12-19\% (Hogan and Brainard, 2015). An obstruction of the brachial artery will lead to paresis or paralysis of the affected thoracic leg. Tetraparesis has been reported as well (Bowles et al., 2010). Clinical signs might be different with embolization sites other than the distal aorta, the communal iliacal arteries, or the brachial artery and are dependent on the secondary affected and ischemic organs. The majority of cats with an ATE event that are presented in practice have no known history of cardiomyopathy. Severe pain and often paralysis of the pelvic limbs are often the first clinical signs noticed. Its dramatic presentation and the lack of preceding clinical signs make ATE one of the most distressing conditions encountered in feline practice (Fuentes, 2012).

\section{PHYSICAL EXAMINATION}

Besides pain and distress often in combination with vocalization, dyspnea and tachypnea, the clinical signs are associated with the affected site of ob- 
struction. As the trifurcation of the distal aorta is the most affected site, representing $71 \%$ of the cases in one large study, paresis or paralysis of either or both hind legs due to a so called "saddle thrombus" are most commonly seen. Studies have shown that only one limb may be affected in up to $26 \%$ of cats with ATE (Smith et al., 2003) (Figure 3). The diagnosis of saddle thrombus can be made from the physical examination alone, based on the following signs: pain, paralysis/paresis of the hind limbs, pulselessness, pallor and poikilothermy, also referred to as the 5 P's. The combination of pelvic limb lower motor neuron signs with absent femoral pulses and cold extremities is pathognomonic for ATE and allows differentiation with a neurological cause. Pulselessness might be hard to differentiate in the front legs, but in addition to cold extremities, the foot pads of the affected $\operatorname{limb}(\mathrm{s})$ are often pale or cyanotic (Figure 4). If the embolized thrombus is small, rapidly lyzed or collateral function is quickly re-established, motor function may be present, and foot pads may be well-perfused by the time the cat is presented. The last $\mathrm{P}$, namely the poikilothermy, refers to the fact that the majority (up to $72 \%$ ) of ATE cats are presented with a reduced rectal temperature, which is considered to be a poor prognostic parameter when it is below $37.2^{\circ} \mathrm{C}$ (Smith et al., 2003; Borgeat et al., 2014). The rectal tone and urinary bladder function are typically maintained.

Furthermore, signs of CHF, e.g. dyspnea and tachypnea, might be present. It is vital to recognize the presence of possible concomitant heart failure, as this will influence the further medical treatment, management and prognosis. Congestive heart failure has been reported in $44 \%$ to $66 \%$ of cases (Smith and Tobias, 2004). An increased respiration rate should not automatically be interpreted as diagnostic of CHF, since the animal may be tachypneic only due to severe pain. Re-assessment after adequate analgesia is necessary in this case. On the contrary, the presence of crackles, a murmur, gallop sound or arrhythmia detected during thoracic auscultation are suggestive for CHF and possible pulmonary edema. However, in $40 \%$ of the cats presented with ATE, auscultation is unremarkable, despite the fact that myocardial disease is the underlying cause in the vast majority of cases (Laste and Harpster 1995). Therefore, underlying heart disease based on a normal auscultation cannot be ruled out (Wagner et al., 2010).

\section{DIFFERENTIAL DIAGNOSIS AND DIAGNOS- TIC TOOLS}

Differential causes of acute posterior paresis include: trauma, intervertebral disc extrusion, spinal lymphoma and other neoplasias, fibracartilaginous embolism-induced infarction (Volk et al., 2011). Acute forelimb paresis can be caused by trauma, foreign body, brachial plexus avulsion.

It is important to emphasize that administration of adequate pain medication (usually opioids), oxygen supplementation in case of dyspnea and reducing stress to an essential minimum are vital parts of the first crucial management step and precedes any further diagnostic tests.

\section{Thoracic radiographs}

Thoracic radiographs are currently the most reliable diagnostic test in order to confirm or exclude the presence of $\mathrm{CHF}$, especially if thoracic auscultation was unremarkable; moreover, they rule out other possible, pulmonary pathologies, which might cause tachypnea or dyspnea. However, in one study, about $11 \%$ of cats with thromboembolism had normal cardiac silhouettes (Laste and Harpster, 1995). In cats with respiratory distress, thoracic radiographs should be postponed until the patient is stabilized, as manipulation might cause further (sudden) clinical deterioration.

\section{Echocardiography}

Once the patient is clinically stable, an echocardiographic examination is advised in order to diagnose a possible underlying heart disease. The vast majority of these patients have a dilated left atrium, possibly in combination with spontaneous echogenic contrast or 'smoke' visible in the left atrial lumen (Figure 2). 'Smoke' indicates an increased risk of embolic stroke in human patients, and is believed to be associated with an increased risk of ATE in cats (Schober and Maerz, 2006). However, a small subset of cats presented with ATE do not have cardiac abnormalities on echocardiography.

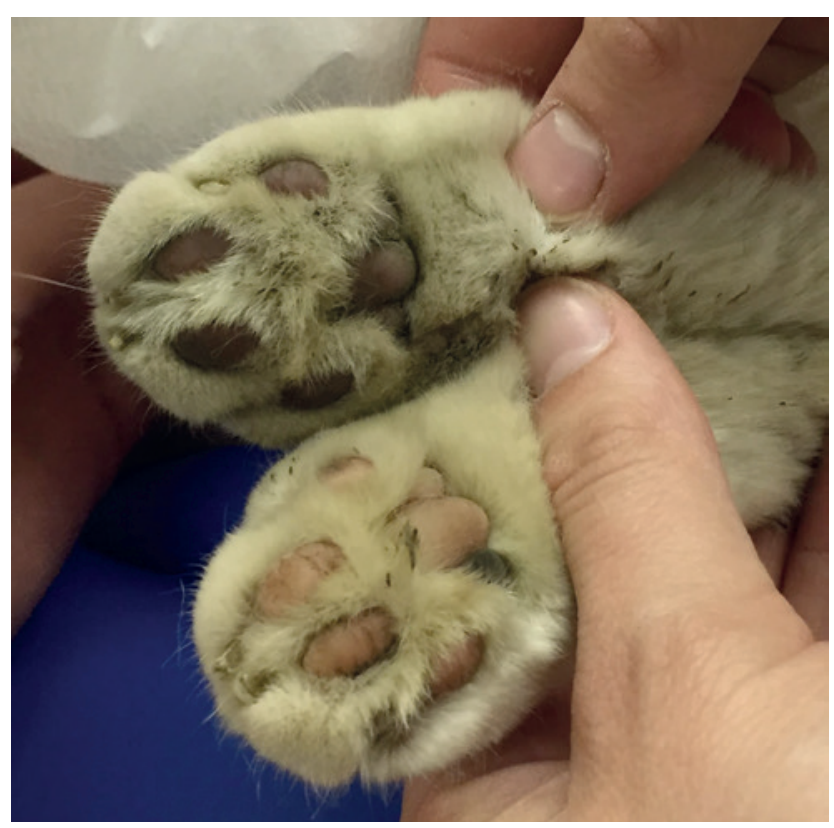

Figure 4. Cyanotic left hind limb footpad and the pale pink right hind limb footpad in this cat with asymmetric ATE of the distal aorta (Photograph courtesy of $P$. Smets). 
Although the diagnosis of ATE in cats with pelvic limb paresis/paralysis is usually based on the distinctive clinical findings rather than imaging, it is commonly possible to identify the thrombus in the terminal aorta using ultrasound imaging. However, the absence of a visible thrombus does not rule out ATE. Where no underlying cardiac disease is identified, further local imaging of the occluded artery is more justifiable (Fuentes, 2012).

\section{Additional tests}

\section{Biochemistry tests}

Biochemistry tests may show a variety of abnormalities, e.g. azotemia, hyperglycemia and phosphatemia. Azotemia can be either prerenal secondary due to reduced renal perfusion or renal due to thromboembolism of a renal artery. Hyperglycemia is often stress-related. Furthermore, serum creatine kinase is often severely increased due to muscle ischemia. Furthermore, alanine aminotransferase values are often elevated (Smith et al., 2003). Potassium values are variable and cats may actually be hypokalemic on presentation, but may develop hyperkalemia once perfusion has been restored. Finally, hypocalcemia and hypernatremia have also been reported. In older cats, total thyroxine levels should be measured as hyperthyroid cats may be at an increased risk of ATE irrespective of any cardiac changes (Laste and Harpster, 1995). Coagulation tests are frequently normal, although D-dimers may be elevated (Bedard et al., 2007; Fuentes, 2012).

\section{Blood pressure measurement}

Doppler blood pressure assessment may be used to confirm the lack of pulse in the affected limb.

\section{Electrocardiography}

Electrocardiography was shown to be informative in a large retrospective study of cats presenting with ATE, with $85 \%$ of affected cats having ECG changes in heart rate or rhythm. The most common ECG abnormalities included a left ventricular enlargement pattern, with large R-waves in leads I and aVL (39\%), sinus tachycardia (28\%), ventricular premature beats (19\%), supraventricular premature beats (19\%), prolongation of the QRS interval (16\%) and a left atrial enlargement pattern (16\%), with a broad, bifid $\mathrm{P}$ wave in lead II (Laste and Harpster, 1995).

\section{DECISION TO TREAT AND PROGNOSIS}

Despite the fact that these patients are often presented with acute limb paralysis in severe pain and distress, which is often perceived by both the owners and veterinary practitioner as inevitably disastrous, a significant subset of cats may recover and lead goodquality lives. The general prognosis is poor with a survival rate of $33 \%$ to $39 \%$, but several cats may display positive prognostic factors indicative of higher survival rates and better mid- to long-term prognosis. Those positive prognostic factors are the absence of $\mathrm{CHF}$, rectal temperature at the moment of presentation $>37.2^{\circ} \mathrm{C}$, (partial) presence of motor function, and the site of thromboembolism and associated affected limbs or other organs (Schoeman, 1999; Borgeat et al., 2014). Although many of these cats are euthanized upon diagnosis, a correct identification of specific prognostic factors obtained from results of physical examination and further testing, enables the veterinarian to give the owner the best possible advice regarding further treatment, management and prognosis. Despite the generally bad prognosis, a study by Smith et al. (2003) showed that rates of survival to discharge improved over a ten-year period, with $73 \%$ of cats surviving to discharge in the last year of study. As many as $70-80 \%$ of cats with a single limb affected survived to discharge, with up to $90 \%$ survival rates in cats presenting with some motor function. Hypothermia appeared to be one of the most reliable markers of reduced initial survival in this study, with $<50 \%$ chance of survival to discharge in cats with a rectal temperature $<37.2^{\circ} \mathrm{C}$ on presentation.

Pain is severe in the initial 24 hours, but decreases substantially after the first 48 hours. The cranial tibial and gastrocnemius muscles may become firm within ten to twelve hours post-embolization due to ischemic myopathy, and ischemic nerve damage may result in 'dropped hocks' as well as loss of distal sensation. The muscles become softer 24 to 72 hours later. However, in some cats, the degree of firmness increases over the first few days, suggesting poorer perfusion. Perfusion and femoral pulses frequently return within three to five days, although in case of severe tissue ischemia, there is a risk of skin and muscle necrosis (Figure 5). This usually manifests within the first two weeks and may lead to loss of toes or decision for limb amputation in severe cases (Smith et al., 2003). Although not well-quantified, many cats may regain

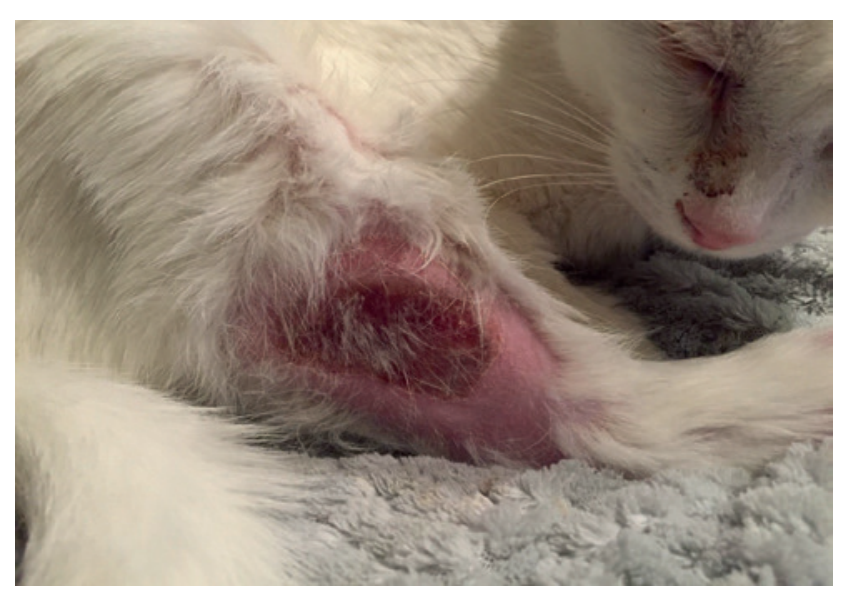

Figure 5. Skin necrosis of the right hind limb, secondary to an ATE event (Photograph courtesy of L. Locquet). 
some to all motor function of the pelvic limbs within four to six weeks from the initial event either due to re-establishment of the collateral vascular network, intrinsic dissolution of the embolus, or recanalization of the obstructed aorta (Smith et al., 2003).

Owners should be aware that their cat might not survive to discharge or might develop a following episode of ATE in the (nearby) future. However, especially cats without $\mathrm{CHF}$ and with only one affected limb, remaining motor function and a rectal temperature above $37.2^{\circ}$ have a substantially better prognosis and may regain partial or complete normal function. Reported ATE recurrence rates range from $24-75 \%$, but because cardiomyopathy is the most prevalent etiological cause, the major cause of death in this subgroup is actually CHF, and not a recurrent episode of ATE (Rush et al., 2000; Lunsford and Mackin, 2007; Payne et al., 2015). Reports of average long-term survival vary between 51 and 350 days (Atkins et al.,1992; Schoeman, 1999).

\section{TREATMENT OF ATE}

\section{Thrombolytic therapies}

Several approaches have been tested in order to remove the obstructing thrombus, but the small size of cats limits minimal invasive embolectomy techniques. Moreover, anesthetic and surgical complications cause high mortality rates. Therefore, this type of treatment is currently contraindicated (Reimer et al., 2006). Medical thrombolytic therapy with streptokinase and tissue plasminogen activator (t-PA) have both been reported in the cat and dissolution of the thrombus has been documented up to 18 hours after initial clinical signs (Moore et al., 2000; Welch et al., 2010). However, sudden reperfusion of ischemic tissues flushing ischemic metabolites, such as potassium and free oxygen species into the bloodstream, leading to reperfusion injury, a life-threatening complication causing arrhythmias, renal dysfunction and acid-base disturbances. This has been reported in $40 \%$ to $70 \%$ of cats receiving thrombolytic therapy and represents the most common cause of death, with survival rates ranging from $0 \%$ to $43 \%$ (Lunsford and Mackin, 2007; Welch et al., 2010). Given these possible lethal complications, thrombolytic therapy should not be administered without thorough consideration of the benefit-to-risk ratio, which should be evaluated for each individual case and its clinical presentation. If available, thrombolytic therapy should be considered in rare cases of cerebral, splanchic or renal infarction, while a cat with a unilateral limb infarction is more likely to achieve complete recovery with conservative therapy (Reimer et al., 2006). The high costs also limit medical thrombolytic therapy in veterinary medicine.

Since thrombolytic therapy is often not possible or even contraindicated, the mainstay of treatment is prevention of continued thrombus formation. The two major categories of antithrombotic drugs are antiplatelet agents and anticoagulants (Fuentes, 2012; Hogan and Brainard, 2015). Doses for these drugs can be found in Table 1 .

\section{Antiplatelet agents}

Antiplatelet agents are currently the mainstay of treatment for feline ATE, because they may improve collateral flow by reducing the amount of vasoactive substances that are released by the platelets (Schaub et al., 1982; Arrebola et al., 2004). Some veterinary cardiologists recommend a combination of two antiplatelet agents (clopidogrel + aspirin) in cats, although there are no studies in cats available about this approach (Fuentes, 2012). This approach is typically limited to those patients that appear to be at particularly increased risk of ATE, i.e. those cats, which continue to have spontaneous echogenic contrast or develop a left atrial thrombus in spite of already receiving therapy (Tan et al., 2010).

\section{Aspirin}

Aspirin irreversibly inhibits the production of thromboxane A2 (TXA2), a potent pro-aggregating and vasoconstrictive molecule. This effect lasts for the lifetime of the platelet. Treating feline patients with aspirin prevent vasoconstriction of collateral circulation. However, cats are still at risk to develop ATE despite treatment, as other TXA2-independent pathways may still result in platelet aggregation (Lunsford and Mackin, 2007). Smith et al. (2003) have shown that the incidence of ATE with low-dose aspirin $(5 \mathrm{mg} /$ cat q72h) compared to high dose aspirin $(40 \mathrm{mg} / \mathrm{cat}$ $\mathrm{q} 72 \mathrm{~h}$ ), was the same. When applying the latter dose, adverse effects are typically gastrointestinal, including anorexia and vomiting secondary to gastric ulceration, and have been reported in up to $22 \%$ of treated cats. Aspirin has been used for the prevention of recurrence of ATE in cats that already had an episode of ATE (secondary prevention) with recurrence rates ranging from $17 \%$ to $75 \%$, and median survival times from 117 to 192 days. However, the recent prospective FATCAT study, evaluating secondary prevention of ATE in cats, has shown aspirin to be inferior to clopidogrel for secondary prevention (Hogan et al.,2015). If monitoring of aspirin therapy is desired, platelet aggregation should be performed before and after at least two weeks of therapy to determine the degree of platelet inhibition. However, it should be mentioned that only a minority of institutions offer this aforementioned test.

\section{Clopidogrel}

Clopidogrel is a specific and irreversible ADP receptor antagonist, as well as a direct platelet antagonist, inhibiting both primary and secondary platelet aggregation. Additionally, clopidogrel also has been 
shown to impair the platelet release reaction and to have vasomodulating effects, maintaining collateral circulation parallel to the occluded artery. Adverse effects are rare, although there are empirical reports of sporadic hypersalivation due to the bitter taste of the drug, vomiting and icterus (Hogan et al., 2015). Clopidogrel has been shown to be superior to aspirin for secondary prevention, with a lower ATE recurrence rate of $49 \%$ (versus $75 \%$ ) and a one-year recurrence rate of $36 \%$ (versus $64 \%$ ). Additionally, clopidogrel has been associated with longer median time to event (443 days) than aspirin (192 days) (Hogan et al., 2015). As with aspirin, monitoring of clopidogrel therapy is rarely performed in veterinary medicine but ADP-induced platelet aggregation is considered the gold standard.

\section{Anticoagulants}

Anticoagulants inhibit the coagulation cascade by interfering with formation of one or more active coagulation factors. In feline medicine, they are now less commonly used than anti-platelet agents, but the new classes of drugs, such as factor Xa-inhibitors may hold promise for future use in cats.

\section{Unfractionated heparin}

Heparin inhibits the coagulation process by counteracting thrombin and activate factors XII, XI, I and IX (Lunford and Mackin, 2007). Heparin is administered SC, but if the cat is in shock, the first dose may also be administered IV. Heparin is often used early in the course of feline ATE to reduce extension of the existing thrombus, and risk of hemorrhages appears to be small. Serial measurement of aPTT has historically been used to monitor effect, with desired aPTT 1.5-2.0 times above baseline. However, in one study, it has been shown that aPTT does not correlate well with plasma heparin levels and anti-Xa monitoring may be a better approach (Smith et al., 2004).

\section{Low molecular weight heparins}

Given its longer half-life and more predictable dose response, low molecular weight heparin (LMWH) has largely superseded unfractioned heparin in the prevention of human deep vein thrombosis, enabling the practitioner to adjust the dose more accurately according to the body weight without the need to monitor the coagulation effects (Lunford and Mackin, 2007). Due to the pharmacokinetic profile and positive clinical studies in humans, there has been great interest in the LMWHs for the prevention of ATE in cats (Smith et al., 2004; Vargo et al., 2009). Alwood et al. (2007) compared enoxaparin, dalteparin and unfractrioned heparin in healthy cats. Only unfractionated heparin showed adequate anti-Xa activity. However, the antiXa activity does not always correlate with clinical antithrombotic effects. There is one retrospective study,

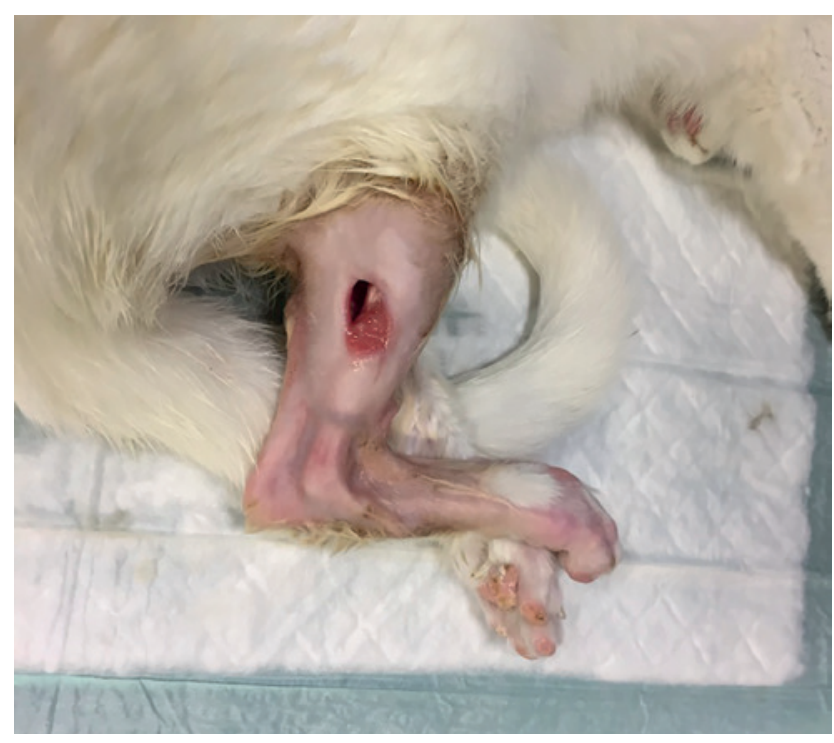

Figure 6. Skin necrosis in the same cat, healing by secondary intention, secondary to an ATE event (Photograph courtesy of L. Locquet).

in which dalteparin was compared to warfarin, and no significant difference in ATE recurrence rate $(43 \%$ versus $24 \%$ respectively) or median survival time (255 days versus 69 days) was demonstrated (Defranscesco et al., 2003). At this timepoint, for the use in the acute in-hospital treatment, there is no clear benefit of LMWH over unfractionated heparin, whereas the cost of the former is much higher.

\section{Warfarin}

Warfarin blocks the effects of vitamin K, necessary for coagulation factors II, VII, IX and X to be activated. However, it is highly protein-bound, has numerous possible interactions with other medications, and has unpredictable pharmacodynamics and pharmacokinetics, with the latter being influenced by both disease state and dietary components. This leads to difficulties associated with its use, safety and effect (Lunsford and Mackin, 2007). Given the unpredictable effect, feline patients exhibit a wide and variable inter-individual and intra-individual anticoagulant response to warfarin, resulting in poor results in studies on warfarin use in cats, with cats with continued thrombogenesis and cats with severe hemorrhages. ATE recurrence rates for cats receiving warfarin range from $24 \%$ to $53 \%$ with estimated mean survival times of 255 days (Defranscesco et al., 2003).

Conclusively, necessary regular monitoring of prothrombin time and corresponding blood sampling in order to assess the effect of warfarin, compromises the life quality of these patients. Although the superiority of warfarin has been demonstrated for the prevention of ATE in humans, the issues with variability in clinical response, requirement for frequent monitoring and bleeding complications have resulted in a rare use of warfarin for thromboprophylaxis in cats. 


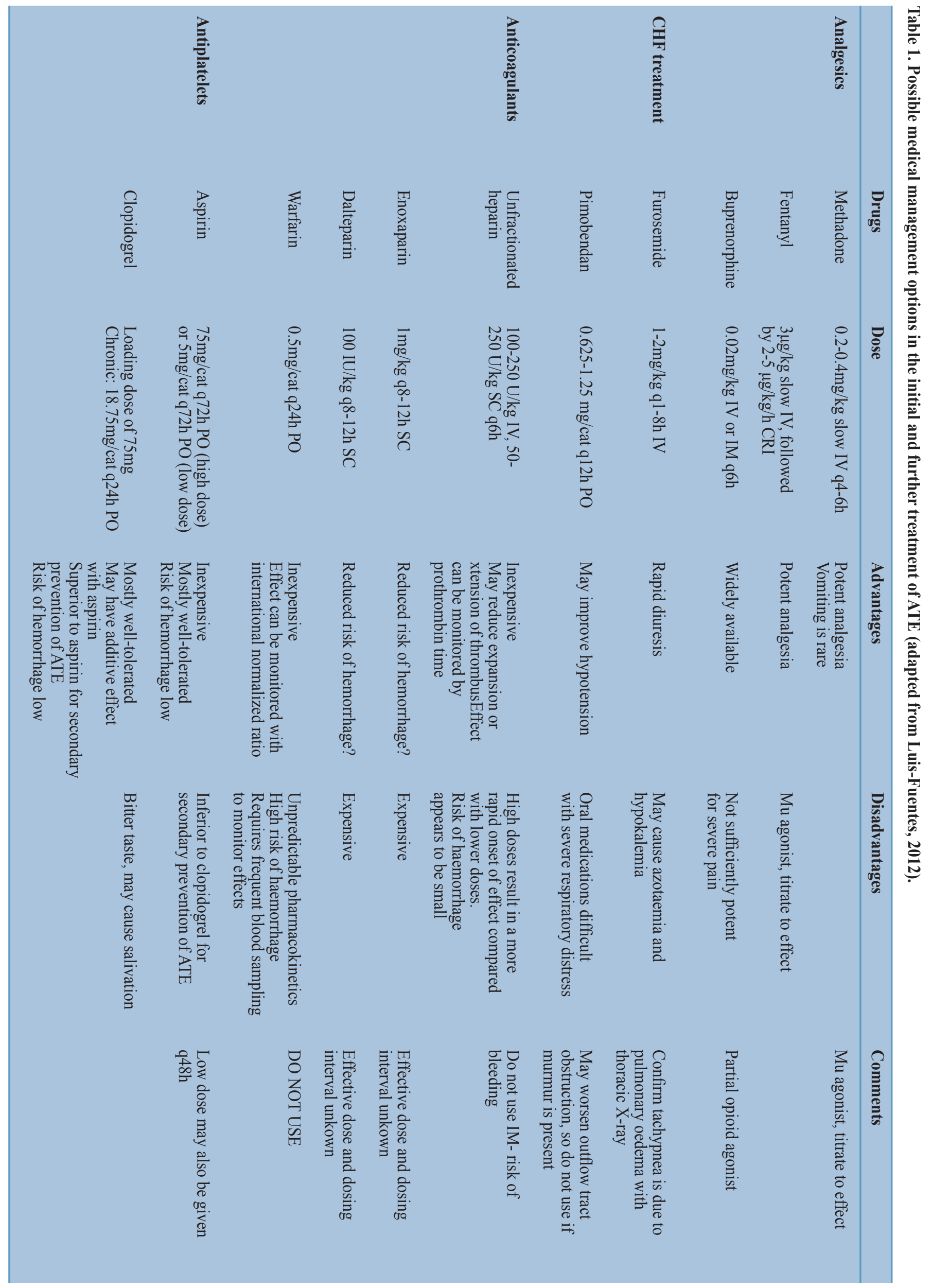




\section{Newer anticoagulants}

Recently, in human medicine, a wide variety of new anticoagulant drugs have been developed to prevent deep venous thrombosis, pulmonary embolism and ATE. Examples of these newly developed anticoagulants are abciximab (a glycoprotein IIb/IIIa antagonist), dabigatran (a direct thrombin inhibitor) and factor Xa-inhibitors, e.g. fondaparinux, rivaroxaban and apixaban. These drugs have been studied thoroughly within human medicine and were designed with excellent efficacy. They exhibit relatively low bleeding risks and generally do not require clinical monitoring. Studies regarding the use of these medications within veterinary medicine are currently lacking. It is expected that factor Xa-inhibitors will likely have a major impact on clinical prophylaxis or ATE in cats. Given the aforementioned characteristics, it would dramatically improve the clinical management of cats at risk of ATE, as current prophylactic protocols are still often based on theoretic effects and benefits, assumed bleeding risk and clinical experience (Hogan, 2017).

\section{General treatment approach}

Currently, a considerable part of these patients are euthanized upon diagnosis, given the sudden onset of devastating clinical signs, often without preceding warning. Given the very painful nature of this condition and due to welfare issues, euthanasia should be discussed and considered indeed, in case no adequate pain relief medication can be provided. Furthermore, failure to receive aspirin, clopidogrel or both is an independent predictor of euthanasia or death within seven days (Borgeat et al., 2014). However, if adequately treated, the majority of these patients are fairly stable within 48-72 hours. Aforementioned prognostic factors, i.e. the presence of $\mathrm{CHF}$, bilateral hind limb paralysis versus only one limb affected, rectal temperature, etc., enable the clinician to have a more substantiated discussion regarding prognosis and expected success rate. Therefore, veterinarians and owners should consider therapy for at least the first 72 hours and not make a decision for immediate euthanasia (Hogan et al., 2015).

Therapy and management of a cardiogenic ATE (CATE) event can be divided in an acute and longterm component. The management of acute CATE includes: 1 . provide pain management, 2 . induction of a hypocoagulable state to reduce continued thrombus formation, 3. improve blood flow to the infarcted arterial bed, 4. treat concurrent $\mathrm{CHF}$ if present, 5. provide supportive care.

This approach does not require extensive financial resources, nor does it necessitate a prolonged hospital stay.

\section{First 60 minutes}

Even before having a thorough discussion with the owners regarding further treatment possibilities, analgesia has to be administered (Figure 7). Opioid analgetics, e.g. methadone, buprenorphine or fentanyl, should be administered at adequate doses and titrated to effect. Furthermore, in case of respiratory distress, oxygen supplementation should be provided without distressing the patient any further. Inspiratory crackles and a gallop rhythm are highly suggestive for the presence of concomitant CHF. If severe pulmonary edema secondary to $\mathrm{CHF}$, is expected, an intravenous/ intramuscular bolus of furosemide should be administered at $1-2 \mathrm{mg} / \mathrm{kg}$ and repeated to effect. The bolus can be repeated hourly or even more frequently, depending on the severity of the respiratory distress and clinical response. Once the cat has been clinically stabilized, thoracic radiographs should be taken in order to confirm the presence of suspected CHF (Fuentes, 2012).

The next priority is to start antithrombotic treatment in order to induce a hypocoagulable state. Given the acute onset of clinical signs, the embolus has often broken off only recently and hence has a 'fresh' surface, which activates and stimulates the coagulation system, resulting in additional thrombus formation and vasoconstriction of the collateral circulation. The basic principle of this antithrombotic treatment is to avoid further extension of the existing thrombus, prevent additional thrombus formation and shift the intrinsic thrombosis/thrombolysis equilibrium toward thrombolysis. Hence, it is important to realize that the main goal is not to lyze the already developed thrombus (Hogan, 2017).

The value of unfractionated heparin or LMWHs for this use has been shown to be quite variable in cats. The recommended dosing regimen for unfractionated heparin is 250 to $375 \mathrm{IU} / \mathrm{kg}$ intravenously initially, followed by 150 to $250 \mathrm{IU} / \mathrm{kg}$ subcutaneously every six to eight hours. Heparin should not be administered intramuscularly because of the risk of injection site hemorrhage. Obtaining a coagulation panel that includes platelet count, PT and aPTT before unfractionated heparin administration is ideal so drug effect can be documented by repetitive re-evaluation of these aforementioned values during heparin therapy. Although not supported by clinical data, prolongation of the aPTT of 1.5 to 2.0 times the baseline value is considered an adequate drug response (Lunsford and Mackin, 2007). As unfractionated heparin is relatively inexpensive and readily available, it is a good choice for acute therapy.

Compared to unfractionated heparin, low molecular weight heparins have similar hemostatic effects; however, the costs are considerably higher. The current recommended protocols for dalteparin in cats 


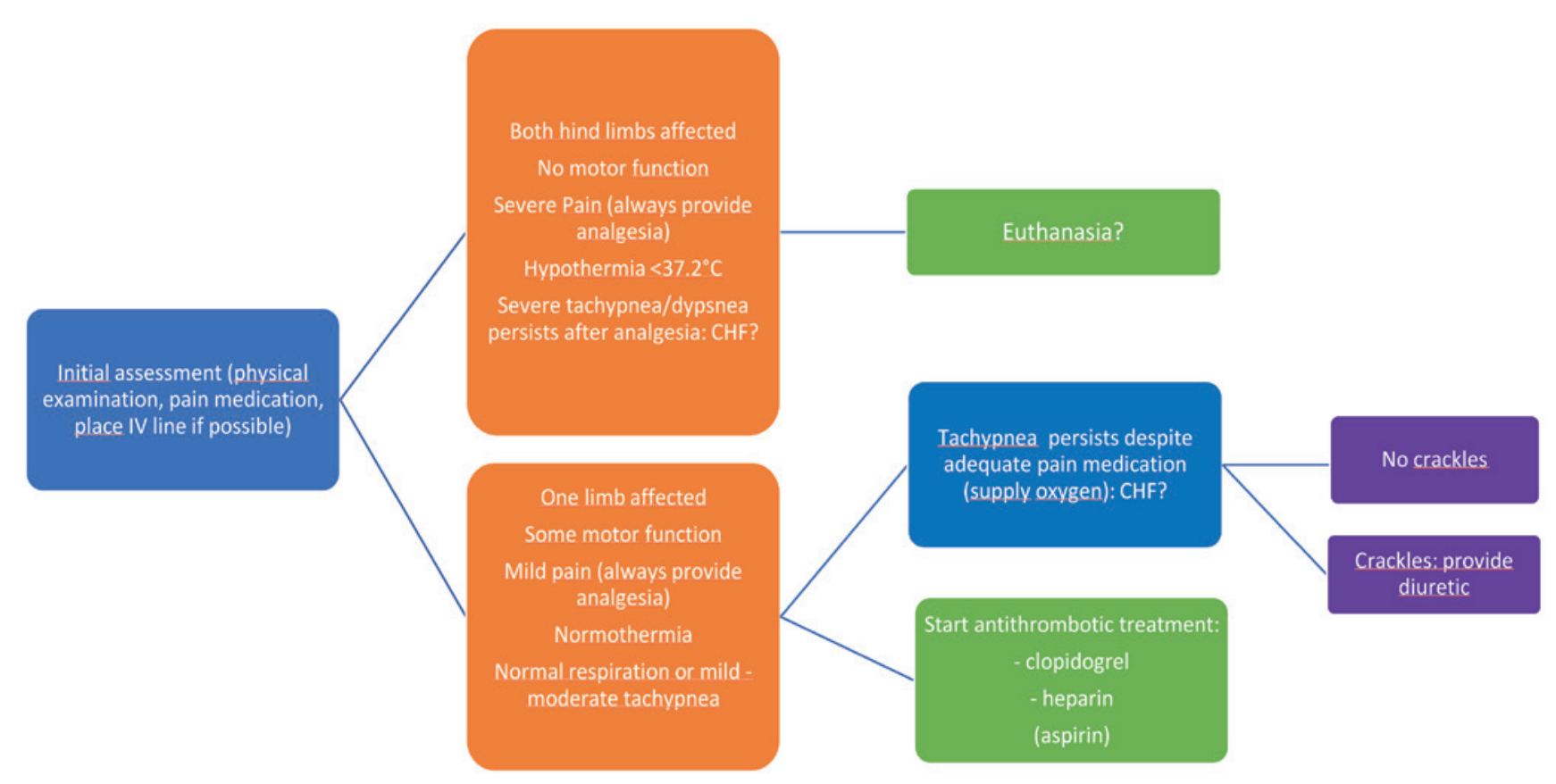

Figure 7. Approach to the cat with ATE: first hour (adapted from Luis-Fuentes, 2012).

are $100 \mathrm{IU} / \mathrm{kg}$ subcutaneously every 24 to 12 hours and for enoxaparin $1.5 \mathrm{mg} / \mathrm{kg}$ SC every 24 to 12 hours, respectively. In a recent pilot study, it has been demonstrated that enoxaparin, when administered at a

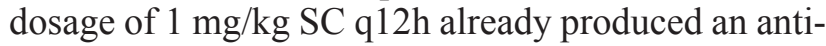
thrombotic effect in a venous stasis model in clinically healthy cats (Van De Wiele et al., 2010). Although they have a beneficial pharmacokinetic profile, allowing them to be administered twice daily, this effect is minimal in the acute management of ATE, so the higher cost of the LMWH may not be justified.

Increasing perfusion to the infarcted region can be attempted by increasing blood flow through the collateral network using antiplatelet drugs, which may be helpful by impairing platelet activation, and hence the vasoactive substances released from platelets. Either aspirin or clopidogrel can be used. Studies have shown that aspirin reduces the amount of released thromboxane A2, which results in an improvement of the collateral flow (Schaub et al., 1982). However, in these studies, very high aspirin doses were used $(150 \mathrm{mg} / \mathrm{kg})$, which might cause toxic adverse effects. Lower doses have not been evaluated with regard to the effect on the collateral network. Similar results have been noted after clopidogrel administration at a dose of $75 \mathrm{mg}$ per cat, due to inhibition of platelet activation and secondary reduction of serotonin release.

An alternative approach is to use oral platelet inhibitors such as aspirin or clopidogrel from the start, and not include heparin at all. Some clinicians use both aspirin and clopidogrel together as early as possible (Fuentes, 2012).

Direct-acting arterial vasodilators such as acepro- mazine have not been shown to have an effect on the collateral network, and are contraindicated as hypotension may result in further reduced perfusion.

\section{First 24 hours}

Further tests and investigations may be undertaken once the cat's pain, ATE and possible CHF have been addressed. Renal values and electrolytes, especially potassium, should be evaluated and an echocardiographic examination should be performed in order to identify possible underlying cardiomyopathy.

Intravenous fluid therapy may assist the treatment of azotemia and the removal of metabolic toxins. However, as a substantial proportion of the patients suffers from CHF, it is recommended to cautiously use parenteral fluid therapy only in cases that would benefit from its use, which is a minority (Fuentes, 2012). Vigilant monitoring of respiratory rate, effort and auscultation for the development of a gallop rhythm is particularly important in these patients. Furthermore, in these particular cases, the diuretic dose should be reduced and other approaches to stimulate the heart function should be assessed.

Given its positive inotropic and lusitropic effect, pimobendan can be administered, although pharmocokinetic and dynamic studies of pimobendan in cats are scarce at the moment and its use is currently not authorized in cats (MacGregor et al., 2011). Pimobendan is not a standard part of the treatment strategy in CATE. Moreover, it may be contraindicated if systolic anterior motion of the mitral valve causing dynamic left ventricular outflow tract obstruction has been 
diagnosed based on the echocardiographic examination. In this case, it may lead to hypotension (Gordon et al., 2012; Gordon and Côté, 2015).

Furthermore, an intensive care setting is essential in the further treatment of ATE cats in order to ensure that these patients are comfortable and early signs of possible complications are detected. Despite the cats being monitored regularly, manipulation should be kept to an essential minimum to prevent any further distress.

\section{4-48 hours post-ATE}

\section{Further investigations/monitoring}

Pain usually decreases and resolves over the first 24-36 hours, with an easily identified improvement, both in general as of the warmth, pulse quality and motor function of the affected $\operatorname{limb}(\mathrm{s})$ in particular (Hogan, 2017). Even without specific thrombolytic measures, the pulse strength will often improve within four to five days. This reflects an improved perfusion of the affected limb(s). Severe ischemic nerve damage may take weeks to improve.

In this particular phase, identifying early warning signs of possible complications or adverse effects is vital, although often challenging. The latter may include CHF, reperfusion injury, azotemia and local consequences of ischemic tissue necrosis. Reperfusion of ischemic tissue may cause fluctuating serum potassium levels, acid-base balance disturbances and elevated reactive oxygen species concentrations, which might be life-threatening. This may cause the patient to deteriorate dramatically and often very sudden, whilst often making apparent good progress before the reperfusion injury (Fuentes, 2012). In patients with ATE, only partially obstructing the affected blood vessel, reperfusion injuries are often less severe. Cardiac and neuromuscular cells are most sensitive for hyperkalemia, resulting in generalized lethargy and bradycardia, possibly evolving towards potential lethal atrial standstill and a comatose state. These can be confirmed with blood tests and electrocardiogram. Hyperkalemia should be treated accordingly with adjusted fluid therapy, glucose, (humilin) insulin, and/ or calcium bicarbonate, dependent on the exact elevated potassium levels and its availability.

\section{Management}

Antithrombotic treatment with clopidogrel, possibly in combination with aspirin, should be continued (Hogan et al., 2015). Despite the fact that most patients will be comfortable after 24 hours, the analgetic treatment should be continued (Table 1). Warm ambient temperatures may stimulate circulation and counteract muscle contraction. Application of heat pads or other external heat sources is contraindicated because of the risk for thermal injury to the infarcted tissues. Furthermore, nutritional support is a critically important aspect that may be overlooked. If the cat is not eating or the caloric intake is inadequate, administration of mirtazapine, an appetite stimulant, or assisted enteral feeding should be considered (Hogan, 2017). Self-mutilation of devitalized limbs occurs in a subset of patients and is characterized by excessive licking or chewing of the toes or hock. Application of a loose fitting bandage barrier is usually effective. Conclusively, it is important to manually void the urinary bladder if necessary, and maintain a clean and comfortable bedding.

\section{$>48$ hours post-ATE}

The vast majority of the cats are more comfortable and happier at home after the initial 72 hours post-ATE. Oral analgetics, e.g. buprenorphine, should be continued at home if there would be still any discomfort present at the time of discharge. The aspirin and clopidogrel can be continued for longer term prophylaxis (Hogan, 2017). Stool softeners, such as lactulose, can be administrated in case of hard stools or constipation. The owner should be informed thoroughly regarding the cat's need for relatively intensive daily home medical care, risk of ATE recurrence, prognosis of the underlying cardiomyopathy and necessity of monitoring the resting respiratory rate, as well as expected costs of treatment and follow-up. Detailed instructions should be given to the owner regarding the physiotherapy and early identification of possible chronic complications from aortic infarction, e.g. self-mutilation, limb necrosis requiring amputation and limb contracture (Fuentes, 2012) (Figures 5 and 6).

Rehabilitation therapy may be instituted after the first two days of ATE and includes gentle passive range of motion, supporting the cat in a standing position to retrain normal placement of the pelvic limbs and feet, and very gentle antegrade massage from the feet to the hip. Owners can be trained how to administer rehabilitation therapy, which can be performed every six hours for the first one to two weeks of recovery, then tapered depending on neuromotor status. In between session, cats are at risk of excoriation of the dorsal aspect of the limbs with affected pelvic limbs as these cats tend to move with the distal limbs extended behind them. The legs hence may require dressings to protect them. Limb edema may be observed days after the event as a consequence of severe muscle injury (and predicts a poorer chance for full recovery) and should not be mistaken for an early sign of ATE (Bonagura, 2010). These complications are usually restricted to cats with very severe ischemic damage and it should be advised to recheck these patients every three to four days by the treating veterinarian to reassess limb function and the need for continued analgesia, presence of pulses, control of CHF signs and the absence of possible necrotic changes in the skin.

Conclusively, cats with underlying cardiomyopathy should be re-evaluated on a regular base via ap- 
propriated diagnostic modalities, e.g. thoracic radiographs and echocardiographic examination, and a therapy adjusted accordingly.

\section{PREVENTION OF ATE}

Given the dramatic presentation and guarded prognosis, prevention of an ATE event is clearly preferable. As already mentioned, cardiomyopathy is the most prevalent etiological cause, and ATE prevention is a standard part of cardiac patients with increased risk. However, cats with non-cardiac diseases predisposing them to ATE, such as hyperthyroidism, protein-losing enteropathy or nephropathy (rare in cats) and pulmonary neoplasia, may also benefit from thromboprophylaxis in specific situations.

\section{Primary prevention}

Primary prevention focuses on preventing an initial ATE event in cats that appear to be at risk; however, at this time, no studies on primary prevention of CATE in veterinary medicine are available. In some studies, it has been tried to define risk factors for the development of CATE. In a retrospective study on cats with $\mathrm{HCM}$, it has been demonstrated that cats presenting with ATE have a significantly larger left atrial size, end-systolic left ventricular diameter and lower fractional shortening than asymptomatic cats or cats with CHF (Payne et al., 2010). Based on this report, some specialists recommend primary prevention in cats with an end-systolic diameter greater than $1.7 \mathrm{~cm}$, a left atrium-to-aortic ratio greater than 2.0 or auricular emptying velocities of $<20 \mathrm{~cm} / \mathrm{s}$. Primary prevention is also indicated in cats with spontaneous contrast or 'smoke' in the left atrium on echocardiography (Schober and Maerz, 2006).

\section{Secondary prevention}

Secondary prevention focuses on preventing a subsequent ATE event in cats that have a history of ATE. In several studies, a recurrence rate of cats receiving some antithrombotic therapy has been reported from $17 \%$ to $75 \%$, with a one-year recurrence rate of $25 \%$ to $50 \%$. The FATCAT study has shown clopidogrel to be superior to aspirin with a ATE recurrence rate of $49 \%$ (versus $75 \%$ for aspirin) and a one-year recurrence rate of $36 \%$ (versus $64 \%$ for aspirin). Clopidogrel was also associated with longer median time to ATE event (443 days) than aspirin (192 days) (Hogan et al., 2015). Cats that have already experienced an ATE event have an objectively demonstrated increased risk, so prevention is recommended in all cats with an objective or suspected history of ATE.

\section{CONCLUSION}

Feline arterial thromboembolism is thought to be an uncommon disease, with a prevalence ranging from $0.3-0.6 \%$ in practice (Smith et al., 2003; Borgeat et al., 2014). Despite the low prevalence, it is considered to be one of the most distressing conditions encountered in feline practice, because of the lack of preceding signs, the devastating clinical presentation and the generally poor prognosis. Given these conditions, the majority of these affected cats are currently euthanized at the time of diagnosis. Although the clinical signs of CATE are dramatic and acute survival is relatively low, the majority of these cats are fairly stable within 48-72 hours. Therefore, owners should consider therapy for at least the first 72 hours and not make a decision for immediate euthanasia (Hogan, 2017). Despite the generally poor prognosis, with survival rates ranging from $33 \%$ to $39 \%$, a subpopulation of these patients may have long-term survival. Some cats survive over a year with a satisfying quality of life, in case of adequate treatment and follow-up. Affected cats may display positive prognostic factors indicative of higher survival rate and better mid- to long-term prognosis, namely the absence of $\mathrm{CHF}$, rectal temperature at the moment of presentation $>37.2^{\circ} \mathrm{C}$, (partial) presence of motor function, and the site of thromboembolism and associated affected limbs or other organs (Schoeman, 1999; Borgeat et al., 2014). A correct identification of specific prognostic factors obtained from results of physical examination and further testing, enables the veterinarian to give the owner the best possible advice regarding further treatment, management and prognosis. Current treatment should always consist of initial clinical stabilization with adequate and adjusted analgetic and antithrombotic treatment.

Furthermore, diuretic therapy should be administered if concomitant CHF is diagnosed. At the moment, there is no fixed treatment protocol, several drug classes can be considered and treatment approach should always be evaluated individually. Prospective studies regarding the primary prevention of ATE events are currently lacking in feline medicine, but for secondary prevention, clopidogrel has been shown superior to aspirin (Hogan et al., 2015). Conclusively, further studies are needed to investigate possible alternative therapies and preventive measurements.

\section{REFERENCES}

The full reference list may be obtained from the lead author on request. 